\title{
Die Blechtrommel in Polen: Der mühsame Weg zum Erfolg
}

\author{
Mirostaw Ossowski
}

\begin{abstract}
The famous novel by Günter Grass, The Tin Drum was translated into Polish in the years of 1968-69, however, its first official edition appeared no sooner than in 1983. Four years earlier the novel had been published by an underground company. This fact shows how serious the obstacles on the road to the novel's reception were. The article presents the road of the novel to the Polish recipient from 1958 until the 1980 s, i.e. from the first trip of the writer to Warsaw and Gdańsk after the war up to the time when the work of the later Nobel Prize winner became the subject of scientific symposiums and monographs. It deals with the Polish connections of the writer which proved to be important for the perception of The Tin Drum as well as the controversy and polemics connected with the novel in the literary press. The article discusses the plans for filming the novel by the Polish director Andrzej Wajda and the reflection of Sławomir Błaut on his work on the translation. The article also discusses the image of Poland and Poles in the novel and how this affected its reception in Poland.
\end{abstract}

Es lässt sich in Polen kaum ein anderes Werk der deutschen Literatur nennen, das sich so mühsam den Weg in die Öffentlichkeit bahnte und doch einen durchschlagenden Erfolg erlebte wie Die Blechtrommel. Bis der Roman 1983 in einem offiziellen Verlag erschien, wurden langjährige Bemühungen um seine Publikation unternommen, die durch die Kontroverse um das Werk und die Eingriffe der Zensur verhindert wurde. Erst nachdem der Roman von dem im Untergrund wirkenden Verlag NOWA 1979 herausgegeben worden war, brachte ihn auch der staatliche Verlag Państwowy Instytut Wydawniczy auf den Markt.

Dabei waren die Anfänge der Rezeption in Polen verheißungsvoll. Die polnischen Leser konnten einen Ausschnitt aus der Blechtrommel kennenlernen, schon bevor der Roman in Deutschland herauskam. In der letzten Nummer der Wochenschrift Polityka im Jahre $195^{8}$ wurde das Kapitel „Fernwirkender Gesang vom Stockturm aus gesungen“ abgedruckt, das von den Besuchen Oskars in dem Spielzeuggeschäft des jüdischen Händlers Sigismund Markus

(C) MIROSŁAW OSSOWSKI, 2016 | DOI 10.1163/9789004291898_005

This is an open access chapter distributed under the terms of the CC-BY-NC License. 
und von den allwöchentlichen Begegnungen seiner Mutter mit ihrem polnischen Cousin Jan Bronski erzählt. Es ist eine der wenigen Romanszenen, die im historischen Stadtzentrum spielen, und sie zeigt den Charme Danzigs in der Vorkriegszeit. Der Text wurde von der angesehenen Übersetzerin Teresa Jętkiewicz ins Polnische übertragen und von dem Literaturkritiker Andrzej Wirth abgedruckt. In einer beigefügten biographischen Notiz über Grass charakterisierte Wirth den bereits mit dem Preis der Gruppe 47 ausgezeichneten Roman als ein hervorragendes literarisches Werk und ein Zeugnis des herzlichen Interesses des Autors „für unser Land“.. Ein halbes Jahr später ließ er in der von ihm herausgegebenen Zeitschrift Nowa Kultura das Anfangskapitel aus der Blechtrommel abdrucken, das er ebenfalls mit einer biographischen Notiz versah. ${ }^{2}$ Am Anfang des Jahres 1960 stellte Wirth den deutschen Autor auch als Lyriker vor, indem er in Nowa Kultura acht kurze Gedichte in eigener Übersetzung publizierte. Er druckte im 19. Heft derselben Zeitschrift auch das Kapitel „Die Tribüne“ ab. ${ }^{3}$ Diese in Polen erschienenen, treffend ausgewählten Auszüge gehören zu den markantesten Passagen des Romans, denn sie zeigen in realistischen und zugleich fantastischen Bildern das Vorkriegsdanzig sowie die Kaschubei als für die Deutschen und Polen bedeutsame Erinnerungsorte. Sie zeugen auch von der unerschöpflichen Vorstellungskraft sowie der sprachlichen Kreativität des Autors.

Der Erstdruck eines Auszugs aus dem bald berühmten Roman in Polen ist auf eine Reise des Autors im Frühjahr $195^{8}$ nach Warschau und Danzig zurückzuführen. Offensichtlich hat sich Grass auch in den Folgejahren bei seinen Gastgebern für die offiziellen Einladungen nach Polen mit seinen Texten revanchiert. Interessanterweise enthält der Vertrag zwischen Günter Grass und dem Luchterhand-Verlag vom 24./25. Februar 1959 über Die Blechtrommel die Klausel, dass die polnischen Rechte für den Roman beim Verfasser bleiben. ${ }^{4}$ Dies ist darin die einzige Ausnahmeregelung hinsichtlich der Übersetzungsrechte, denn sonst obliegt nach diesem Vertrag die Vergebung von Nachdrucklizenzen für Die Blechtrommel den beiden Vertragspartnern, dem Autor und dem Verlag. Wohl dank dieser Klausel konnte Grass auch den Druck des Romans durch den Untergrundverlag NowA 1979 genehmigen, ohne dass dadurch die Urheberrechte des Luchterhand-Verlags verletzt wurden.

1 Grass (1958). Übersetzung sämtlicher Zitate aus dem Polnischen von M.O.

2 Grass (1959).

3 Grass (1960). Dazu eine biographische Information von Andrzej Wirth und eine Zeichnung von Barbara Jonscher, die Oskar mit seiner Trommel darstellt.

4 Akademie der Künste, im Folgenden: (AdK), Berlin, Günter-Grass-Archiv, Nr. 10197. 
Die Rezeption der deutschen Belletristik in Polen von 1945 bis zu der demokratischen Wende 1989 ist als ein Politikum zu betrachten. ${ }^{5}$ In der Öffentlichkeit herrschte ein ideologisch begründetes Misstrauen gegenüber dem Westen und das Verhältnis zu den Deutschen war zusätzlich durch den Schatten des Zweiten Weltkrieges belastet. Nach dem Umbruch des Jahres 1956, als die stalinistische Epoche zu Ende ging, öffneten sich die polnischen Verlage gen Westen. Es erschienen in den nächsten Jahren die Übersetzungen von Autoren wie Albert Camus, Friedrich Dürrenmatt, Max Frisch, Ernest Hemingway u. a. Die Leser konnten nun auch bisher kaum bekannte westdeutsche Autoren kennenlernen wie Ernst Wiechert, Heinrich Böll und eben Günter Grass. In einer Zeit, wo zwischen Polen und der Bundesrepublik Deutschland weder diplomatische noch wirtschaftliche Beziehungen bestanden, ebneten die Kulturschaffenden den Weg für die künftigen bilateralen Kontakte.

Auch Grass fiel in diesem langwierigen Annäherungsprozess eine gewichtige Rolle zu. Sein Geburtsort im deutsch-polnischen Kulturgrenzraum und selbst seine familiären Verhältnisse prädestinierten ihn zum Mittler zwischen Deutschen und Polen. Danzig, eine im 20. Jahrhundert im vielfachen Sinne symbolträchtige Stadt, war in der deutschen Kultur tief verwurzelt, hatte aber über Jahrhunderte hinweg auch enge politische und wirtschaftliche Beziehungen mit Polen. In der Zwischenkriegszeit eine Freie Stadt unter dem Schutz des Völkerbundes, wurde Danzig am 1. September 1939 an das Dritte Reich angeschlossen und seit 1945 gehört es zu Polen. Während seiner ersten Reise nach dem Krieg nach Danzig konnte der Schriftsteller unschätzbare Informationen für seinen Erstlingsroman sammeln. Der bedeutendste, wenn auch nicht der einzige Ertrag war das Romankapitel über die Polnische Post in der Freien Stadt Danzig, wo vor dem Krieg der Cousin seiner Mutter, Franciszek (Franz) Krause, gearbeitet hatte, der zu den Verteidigern der Post am 1. September 1939 gehörte und der danach mit anderen Postbeamten standrechtlich erschossen wurde. Grass fand zwei überlebende Postbeamte, die ihm Auskünfte über den Kampf um das Postgebäude erteilten. Ihr Bericht war „so absurd wie ebenfalls wahrscheinlich, dass ich ihn teilweise im entsprechenden Kapitel der Blechtrommel benutzen konnte“, erinnerte sich der Autor nach Jahren. ${ }^{6}$ Die beiden Augenzeugen erzählten ihm von dem Ablauf der Kämpfe, der Stimmung in dem umzingelten Postgebäude und der Flucht einiger Verteidiger, was er in seinem Roman auch wiedergab. Man hat ihm später die Diffamierung der Postverteidiger zum Vorwurf gemacht, während in Polen heroisierende Darstellungen in Film und Publizistik verbreitet wurden.

5 Vgl. Krauss (2011) 94.

6 Karaś (2009). 
Im Jahre 1962 erschien in der regionalen Zeitschrift Pomorze zum ersten Mal auch ein umfassender Auszug aus der Blechtrommel, der von der Verteidigung der polnischen Post handelte. ${ }^{7}$ Der Übersetzer, Bolesław Fac, bezeichnete im beiliegenden Text den Roman als ein scheinbar surrealistisches und absurdes, in Wirklichkeit aber durchaus realistisches Werk. Er nannte Grass „einen westdeutschen Autor polnischer Herkunft (von einer kaschubischen Mutter)“ und verglich sein Talent mit dem von Thomas Mann. Im Jahre 1963 brachte der Verlag Czytelnik das erste vollständige Werk von Grass in Polen, die Novelle Katz und Maus, heraus. Zugleich begann in den polnischen Zeitschriften die Kontroverse um den Schriftsteller. In kurzem Abstand traten zwei namhafte Schriftsteller und Publizisten, Jan Dobraczyński und Wojciech Żukrowski, mit kritischen Aufsätzen auf. ${ }^{8}$ Der zu dem Kreis der katholischen Intellektuellen gehörende Dobraczyński äußerte ethische Zweifel und entrüstete sich über die Verbindung von erotischen und religiösen Motiven in der Blechtrommel, die er als Gotteslästerung empfand. ${ }^{9}$ Auch bemängelte er die Darstellungen der polnischen Motive, die die westdeutschen Stereotypen von der polnischen Dummheit vervielfältigten, etwa die ,irrsinnige‘ Kavallerie mit ihrem Anführer Pan Kiehot.

Einige lobende Worte fand er dagegen für die mit Talent verfasste Novelle Katz und Maus, die er - ähnlich wie den Erstlingsroman Grass' - auf Deutsch gelesen hatte, noch bevor die polnische Übersetzung erschienen war. Anders beurteilte die Novelle Wojciech Żukrowski, der dem Autor Pornographie vorwarf und den Verfasser des Vorworts, Andrzej Wirth, kritisierte, der die Novelle gelobt und bei dem Autor die Faszination für das Polentum konstatiert hat. Nach Żukrowski hat Grass die Polen geradewegs als „eine Bande Trottel und Angeber" präsentiert, und wenn er auch die Deutschen keinesfalls im besseren Licht darstelle, so gäbe es keinen Grund, von einer Verbundenheit den Polen gegenüber zu schreiben. Der Autor der Kritik griff auch die Übersetzter Irena und Egon Naganowski an, die angeblich die Namen für männliche Geschlechtsorgane um poetische und kräftige Ausdrücke bereichert hätten.10 Er hielt Katz und Maus für künstlerisch schwach, betrachtete dagegen - anders als Dobraczyński - Die Blechtrommel als einen „preisgekrönten [...] ein wenig unbequemen Roman“, den man mit einem "sinnvollen Kommentar“ herausgeben sollte. Nach Jaroszewski konnte man sich aufgrund der Rezensionen von Dobraczyński und Żukrowski, trotz des unterschiedlichen Tenors, die Meinung

\footnotetext{
$7 \quad$ Grass (1962a).

8 Die Kontroverse beschreibt ausführlich Jaroszewski (2002); vgl. auch Żyliński (2009).

9 Dobraczyński (1963).

10 Żukrowski (1963).
} 
bilden, die beiden Werke „seien in konfessioneller, ethischer, politischer und künstlerischer Hinsicht für den polnischen Leser völlig ungeeignet, denn als Bücher von minderwertiger Qualität verletzten sie dessen religiöse, moralische und patriotische Gefühle ${ }^{\text {"11 }}$.

Einen beachtenswerten Nebenschauplatz dieser Kontroverse stellt der Abdruck der Auszüge aus der Blechtrommel in der in Paris herausgegebenen polnischen Exilmonatsschrift Kultura im April 1962 dar. $^{12}$ Wenige Monate zuvor wurde der Roman in dieser Zeitschrift von Konstanty Jeleński rezensiert, der in seiner Besprechung besonders auf die polnischen Aspekte des Romans verwies und konstatierte: „Günter Grass könnte ein polnischer Schriftsteller sein: diese Tonart der fantastischen Autobiographie, Poesie und des schwarzen Humors kennen wir von Gombrowicz, von Schulz. ${ }^{13}$ Eine Publikation der Romanauszüge in der Pariser Kultura brachte den Autor in die Opposition gegenüber den politischen Machthabern in Polen und konnte damals als Verrat aufgefasst werden. ${ }^{14}$ Für die Autoren der kritischen Aufsätze war dies gewiss keine Empfehlung. Dobraczyński brachte seine Antipathie auch wortwörtlich zum Ausdruck: „Es verwundert nicht, dass das Buch Grass' heiße Lobsprüche in der Pariser Kultura erntete. ${ }^{15}$

Einen wenig bekannten Aspekt dieser Kontroverse beleuchtete Jahre später Andrzej Wajda, den Günter Grass seit 1962 als Regisseur für die Verfilmung von Katz und Maus und später für die Blechtrommel und auch andere Werke zu gewinnen versuchte. Wajda zeigte Interesse an Katz und Maus, zog sich dann aber von dem Projekt zurück; den Film hat Hansjürgen Pohland mit Verzögerung im Jahr 1966 in Danzig gedreht. Wajda begründete seine Absage mit den Dreharbeiten an einem anderen Film und mit Zeitmangel, in Wirklichkeit aber hatte er von den Behörden keine Genehmigung für die Mitwirkung an der Verfilmung bekommen. Der Regisseur äußerte 1999: „Günter Grass [...] hatte seine Novelle Katz und Maus für mich bestimmt. Leider hielten es die politischen Behörden für unmöglich, dass ein polnischer Regisseur einen deutschen Film mit deutschen Schauspielern macht, dessen Handlung in der Ostseeregion, also in Polen spielt. Die Rezension von Wojciech Żukrowski hat diesem Plan

\footnotetext{
$11 \quad$ Jaroszewski (2002) 82-83.

12 Grass (1962b).

13 Jeleński (1962) 217.

14 Dieser Aspekt der polnischen Grass-Rezeption, die Publikation in der Pariser Kultura, wurde nicht von allen Forschern der Grass-Rezeption in Polen berücksichtigt. Auf den Zusammenhang mit der Kontroverse um Grass verweist jedoch Zielińska (2006).

Dobraczyński (1963) 2.
} 
viel geschadet. Es wurde eine Intrige geschmiedet, um meine Mitarbeit an dem Film zu verhindern. “16

In den sechziger Jahren begann die internationale Karriere von Andrzej Wajda. Der polnische Regisseur drehte 1962 den Film Sibirska Ledi Magbet in Jugoslawien und 1967 den britisch-deutschen Film Gates to Paradise/Die Pforten des Paradieses. Er schuf mit Leidenschaft Filme für das polnische Publikum, die Arbeit im Ausland passte ihm wegen der sprachlichen Barriere und der für ihn ungewohnten Arbeitsbedingungen jedoch weniger. Er nahm aber hin und wieder die Angebote der ausländischen Produzenten an. Es ist interessant, dass Wajda und Grass seit 1963 im Briefwechsel standen und sich über die Verfilmung der Blechtrommel austauschten. Der Regisseur hatte den Roman aus der noch nicht publizierten Übersetzung kennengelernt und einen Entwurf für die Verfilmung ausgearbeitet. Intensiv waren ihre Kontakte 1973, als Wajda Grass sein Konzept vorlegte. ${ }^{17}$ Er wollte ausgewählte Szenen aus allen drei Büchern zeigen, das dritte Buch sollte dabei im Vordergrund stehen, das sich nach Wajda zu jener Zeit größten Interesses erfreute. Darin besteht ein gravierender Unterschied zwischen seinem Konzept und der späteren Verfilmung von Volker Schlöndorff, der sich auf die ersten zwei Bücher beschränkte und damit dem internationalen Publikum Danzig als einen Erinnerungsort von besonderem Gewicht im deutsch-polnischen Grenzraum zeigte. Wajda, der in einer anderen politischen Realität lebte und als Regisseur wirkte, konnte sich eine solche künstlerische Vision nicht erlauben. Der Film sollte nach seiner Konzeption mit den Bildern aus dem Fotoalbum beginnen, darunter auch mit dem abgebildeten weiten Rock der Großmutter, dann sollte Oskar mit der Blechtrommel erscheinen und seine Lebensgeschichte erzählen. Der polnische Regisseur wollte in dieser Rolle Roman Polański besetzen, der auch grundsätzlich dazu bereit war. Wajda rechnete damit, dass Polański als erfahrener Regisseur ihm helfen würde, die technischen Probleme bei der Darstellung Oskars zu überwinden. Die drei großen Namen, Grass, Wajda und Polański, bewirkten, das die Studio Hamburg Produktionsgruppe bereit war, das Projekt zu realisieren. Dass es letztendlich nicht dazu kam, lag an der Zögerung Wajdas, den Vertrag zu unterzeichnen, möglicherweise auch deshalb, weil damals kein passendes Drehbuch vorlag. ${ }^{18}$

Die Kontroverse um Die Blechtrommel wurde in der polnischen Publizistik bis in die neunziger Jahre fortgesetzt. Zum Teil führten die Gegner des Romans dieselben Argumente an, die bereits in den Aufsätzen von 1963 formuliert

\footnotetext{
$16 \quad$ N.N. (1999) 28.

17 Wajda an Grass, 16.05.1973, (AdK), Berlin, Günter-Grass-Archiv, Nr. 9539.

18 Mehr zu den geplanten Verfilmungen der Werke von Grass durch Wajda: Ossowski (2013).
} 
worden waren. So argumentierte Michał Misiorny 1977 scheinheilig in der Parteizeitung Trybuna Ludu, dass das Werk in Polen aus Rücksicht auf die religiösen Gefühle der Katholiken und weil er die Normen des sozialistischen Humanismus verletzte nicht erschienen sei. Nach der Veröffentlichung der Blechtrommel führte derselbe Publizist 1984 neben den alten Argumenten gegen den Roman ein neues an: die Darstellung von einigen Aspekten der Befreiung Danzigs $1945 .{ }^{19}$ Auch der in dieser Stadt vor dem Krieg aufgewachsene polnische Schriftsteller Brunon Zwarra, Jahrgang 1919, übte über Jahrzehnte scharfe Kritik an dem Roman, da darin die Verteidigung der Polnischen Post falsch dargestellt und die Polen diffamiert würden. ${ }^{20}$

In den polnischen Zeitungen und Zeitschriften wurden jedoch ebenfalls Artikel publiziert, deren Autoren den künstlerischen Wert der Blechtrommel hervorhoben und deren Veröffentlichung postulierten. 1971 erschien in der Zeitschrift Polityka der ursprünglich für die Buchausgabe der Blechtrommel als Vorwort bestimmte Text von Roman Bratny, der den Romanautor verteidigte und gegen die Vorwürfe der Pornographie und Gotteslästerung polemisierte. ${ }^{21}$ Auch die Redaktion der Monatsschrift Literatura na świecie [Weltliteratur] hat für Grass eine Lanze gebrochen. Sie sprach sich für die Veröffentlichung solcher Werke der Weltliteratur aus, deren Mangel man in Polen besonders schmerzlich empfand; dazu wurden auch die Texte von Günter Grass gezählt. ${ }^{22}$ Jan Koprowski verteidigte in dieser Zeitschrift in einer Rezension über Aus dem Tagebuch einer Schnecke den Roman Die Blechtrommel, indem er meinte, dass Grass eine Obsession für Polen habe und wie kaum ein anderer deutscher Schriftsteller die polnischen Angelegenheiten kenne. ${ }^{23}$ Bereits 1963 hat Koprowski im ähnlichen Ton auf den kritischen Aufsatz von Wojciech Żukrowski geantwortet und gefordert, in der Blechtrommel die Bilder und Fakten nicht aus dem Kontext und der Aura des Romans herauszugreifen. ${ }^{24}$

Literatura na świecie veröffentlichte 1976 zehn von den sechzehn Kapiteln aus dem ersten Buch der Blechtrommel unter dem Titel „Zasnąłem pod czterema spódnicami“ [Ich schlief unter den vier Röcken ein]. ${ }^{25}$ Es war der umfassendste publizierte Teil des in Polen noch nicht erschienenen Romans.

19 Misiorny (1977); Misiorny (1984). Vgl. auch Jaroszewski (2002) 87-88.

20 Vgl. Zwarra (1984) 357-358, 619. Zwarra meinte irrtümlich, dass sich Grass auf die 1939 in Danzig erschienene Publikation von Artur Bassarek Danzigs Befreiung. Ein Tatsachenbericht mit Bildern stützte.

21 Bratny (1971).

22 Hertz (1973). Vgl. auch Zielińska (2006) 68-69.

23 Koprowski (1971).

24 Koprowski (1963).

25 Grass (1976). 
Im Band fanden sich auch drei Aufsätze: eine Studie von Wacław Sadkowski über die polnischen Motive bei Grass, die begeisterte Besprechung des Romans von Hans Magnus Enzensberger aus dem Jahre 1959 und ein Essay von Ralph Freedmann, des Weiteren mehrere Gedichte sowie ein Interview mit Grass von Karol Sauerland. Da in diesem Jahr ebenfalls in anderen Zeitschriften Auszüge aus dem Werk publiziert wurden, erschien dies wie eine Kampagne im Rahmen der offiziellen Kulturpolitik für die Herausgabe des Romans in Polen. Mirosława Zielińska führt diese Aktivitäten auf ein Echo in den polnischen Zeitungen und Zeitschriften zurück, das der Film Teure Geschichte (1975), über den Wiederaufbau der im Zweiten Weltkrieg zerstörten Stadt Danzig, fand, den Volker Petzold für den Fernsehsender NDR gedreht und in dem Günter Grass als Moderator fungiert hatte.

In den 7oer Jahren fängt auch die Rezeption der Blechtrommel und der anderen Werke von Grass in der polnischen Literaturwissenschaft an. Im Jahre 1975 verteidigt in Wrocław Zbigniew Światłowski seine auf Polnisch verfasste Dissertation über die Prosa Grass' in den Jahren 1959-1968. Vier Jahre später legt Henryka Szumowska ihre Doktorarbeit zum Thema ,Günter Grass als politischer Schriftsteller vor. Auch die im Ausland publizierten Werke der Sekundärliteratur erwecken zunehmendes Interesse, wovon ein 1975 in der Zeitschrift Przeglad Humanistyczny publizierter Rezensionsartikel von Teresa Bauer über das in Paris zwei Jahre zuvor erschienene Buch von Robert Leroy ,Die Blechtrommel' von Günter Grass. Eine Interpretation zeugt. Die Autorin der Rezension berichtet ausführlich über die Ausführungen von Leroy, verweist auf die Vielschichtigkeit und Vieldeutigkeit des Romans, weswegen sich Die Blechtrommel einfachen Interpretationen verschießt, und betont zum Schluss, dass Leroy in seiner Auslegung generell über die ,Froschperspektive‘ Oskars nicht hinausgeht. Ein reger Diskurs über den Erstlingsroman Grass' entwickelt sich in den 8oer Jahren, als die polnischen Germanisten zwei Monographien über Grass publizieren: Zbigniew Światłowski ,Gdańska trylogia Güntera Grassa [Danziger Trilogie Günter Grass'] (1982) und Norbert Honsza Günter Grass. Werk und Wirkung (1987). Unter den Polonisten profiliert sich Maria Janion als eine namhafte Grass-Kennerin, die 1981 an der Danziger Universität die erste akademische Debatte des Schriftstellers in Polen und 1985 ein Seminar im Warschauer Studentenklub Hybrydy organisiert, an dem Grass jedoch nicht teilnehmen kann, weil ihm die polnischen Behörden das Visum verweigern. Janion gibt auch einen inhaltsreichen Sammelband Polskie pytania o Grassa [Polnische Fragen zu Grass] (1988) heraus, dessen Bedeutung darin liegt, dass er die polnische Lesart der Werke von Grass breit präsentiert. 1987 findet eine von der Germanistik in Wrocław organisierte Grass-Tagung in Karpacz statt, an der auch der deutsche Schriftsteller teilnimmt. Der Ertrag ist ein von Norbert 
Honsza, Jerzy Łukosz und Marian Szyrocki herausgegebener Sammelband Der Mensch wird an seiner Dummheit sterben. Günter-Grass-Konferenz, Karpacz, 17.-23. Mai 1987.

Die 8oer Jahre sind wohl ein Höhepunkt in der polnischen Rezeption der Blechtrommel, die endlich auch den Lesern zugänglich ist. Die Vorbereitungen der polnischen Ausgabe begannen im Jahre 1967, als die literarische Agentur Geisenheyner und Crone, die sich auf die Kontakte mit den osteuropäischen Staaten spezialisiert hatte, einen Lizenzvertrag über die polnische Übersetzung der Blechtrommel vermittelte. In dem Vertrag wurde eine Mindestauflage von 20.00o Exemplaren festgelegt. Nach dem Vertragsabschluss schrieb Grass an Ernst W. Geisenheyner: „Eigentlich, - und das sollte man in Polen wissen, - ist Die Blechtrommel ein Roman für zwei Länder, für Polen und für Deutschland [...]. ${ }^{“ 26}$ Der Warschauer Verlag beauftragte nun Sławomir Błaut mit der Aufgabe, den Roman zu übersetzen. Błaut, ein angehender Übersetzer, sah in der Arbeit an dem weltberühmten Roman eine hervorragende Chance auf Erfolg. Er hat seine translatorische Arbeit an dem Text 1968-69 in einem relativ kurzen, aber inhaltsreichen Aufsatz Polak ttumaczy Grassa [Ein Pole übersetzt Grass] geschildert. ${ }^{27}$ Er erzählt darin auch von den Emotionen während der langen Wartezeit auf das Erscheinen des Romans, als er fürchten musste, dass die aufwendige Arbeit an der Übersetzung vielleicht umsonst war. Nach der ersten Lektüre des Romans wurde ihm bewusst, dass er es mit einem Werk von ungewöhnlicher Dichte zu tun hatte, das die handfeste Realität mit suggestiven poetischen Visionen verband. Er hoffte, dass die von ihm übersetzte Blechtrommel bei den polnischen Lesern die Kenntnis der Deutschen und deren Geschichte bereichern würde. Zugleich bewegte ihn die Sorge, ob „Grass' Poetik der Überspitzungen und Absonderlichkeit“28, die auch die polnischen nationalen Symbole erfassen, das Vorstellungsvermögen der Polen ansprechen und bei ihnen auf Verständnis stoßen werde. Denn Błaut war sich dessen bewusst, dass die Literaturkritiker poetologische Zeichen mit historischen Informationen zu verwechseln und zu missverstehen pflegen.

Błaut verweist in seinem Aufsatz auch auf die sprachliche Vehemenz der Blechtrommel und berichtet über die Schwierigkeit während der Arbeit an dem Text, die ihm der lexikalische Reichtum, die Vielfalt der syntaktischen Formen und die Fülle der Metaphern bereiteten. Bei dem Versuch, „in der Wahl der Worte mit dem Autor Schritt zu halten“29, konnte er kleine Erfolge, aber auch

\footnotetext{
26 Grass an Geisenheyner, am 23. Mai 1967. (AdK), Berlin, Günter-Grass-Archiv, Nr. 3035.

27 Vgl. Błaut (1999).

28 Błaut (1999) 219.

29 Błaut (1999) 220.
} 
Niederlagen verzeichnen. Bereits das erste Wort des Textes: „Zugegeben“ erwies sich als eine Hürde und ließ sich im Polnischen nur mit mehreren Vokabeln („Nie będę ukrywał“) wiedergeben. Besondere Probleme hatte der Übersetzer mit dem Wort „zersingen“. Błaut fiel kein Neologismus ein und er verwendete als Äquivalent das im Polnischen eher seltene Wort „rozśpiewać“ [zum Singen bringen], dem er eine neue Funktion zu geben versuchte, indem er das Wort mit der Wendung „rozbijać śpiewem szkło“ [das Glas mit Singen zerschlagen] ankündigte. Es war trotzdem eine wenig glückliche Lösung. Ein anderes Problem, das Grass' Texte dem Übersetzer boten, waren die dialektalen Formen und die Vorstadtmundarten, die jedoch in der Blechtrommel relativ selten sind. Erst in dem Roman Die Hundejahre hatte Błaut größere Probleme, erstens die Bedeutungsebene der Mundarten präzise zu entziffern, zweitens ihre Vielfarbigkeit wiederzugeben. Der Übersetzer vertritt den Standpunkt, dass bei der Übertragung der Mundart eine gewisse Verarmung unvermeidbar sei, da man die mundartlichen Formen nur leicht in der Flexion und der Syntax andeuten könne. Eine zusätzliche Schwierigkeit bot ihm in der Blechtrommel der Skat, ein in Polen nur in wenigen Regionen bekanntes Kartenspiel. Der Übersetzer musste für die Translation die Spielregeln, die Terminologie und die Dramatik dieses Spieles erst kennenlernen.

$\mathrm{Zu}$ Beginn des Jahres 1970 schickte der Chefredakteur des polnischen Verlages, Andrzej Wasilewski, dem Autor die Übersetzung der Blechtrommel und schlug ihm vor, eine Reihe ,kleiner' Streichungen oder Retuschen vorzunehmen. ${ }^{30}$ In Wirklichkeit handelte es sich um diverse Eingriffe der Zensur. Nach der Auffassung von Andrzej Wirth und Karol Sauerland, die Grass um die Begutachtung der Übersetzung und der vom Verlag vorgeschlagenen Kürzungen bat, waren diese zum Teil unvermeidbar, wenn der Roman in Polen herauskommen sollte. ${ }^{31}$ Nach Wirth waren die Streichungen für die Substanz des Romans nicht entscheidend und er schlug vor, mit dem polnischen Verlag zu verhandeln, um einige Stellen noch zu retten. Einen ähnlichen Standpunkt vertrat Sauerland.

Die Eingriffe betrafen zum einem die Rolle der Roten Armee bei der Besetzung Danzigs und die Darstellung ihrer Soldaten. So sollten etwa die Läuse am Kragenrand eines Kalmücken aus dem Text verschwinden, die Russen nicht stehlen und keine Frauen ausprobieren (die Episode im Keller stellte einen

$30 \quad$ G. Grass an Otto F. Walter 21.01.1970, (AdK), Berlin, Günter-Grass-Archiv, Nr. 9924.

$31 \quad$ Andrzej Wirth, Die Blechtrommel. Die Gutachtung der polnischen Übersetzung, Standford University, 26.6.1970 [Masch.-Sch.]; Karol Sauerland, Gutachten der polnischen Übersetzung der Blechtrommel von Günter Gras Wien, 7.09.1970 [Masch.-Sch.], (AdK), Berlin, Günter-Grass-Archiv, Nr. 9895.4. 
Ausnahmefall dar). Der Marschall Rokossowski sollte nicht als der Eroberer und Brandstifter Danzigs gezeigt werden. Zum anderen handelte es sich bei den Streichungen um die Darstellungen der Polen, die sich etwa keinen deutschen Besitz mit Gewalt aneignen und nicht den Tod eines deutschen Sozialdemokraten verursachen sollten. Ein für die Zensoren wunder Punkt waren in der Blechtrommel die Anspielungen auf die Partisanen. Der Grund für die Streichungen - wie Karol Sauerland wohl mit Recht urteilte - war innenpolitischer Natur, denn Ende der 6oer drängte in Polen eine nationalistische Gruppe um Mieczysław Moczar an die Macht, die umgangssprachlich ,Partisanen' genannt wurde. In der polnischen Übersetzung gewann z.B. der folgende Satz ungeahnte neue Konnotationen: „Partisane sind nie zeitweilig Partisane, sondern sind immer und andauernd Partisane, die gestürzte Regierungen in den Sattel heben, und gerade mit Hilfe der Partisane in den Sattel gehobene Regierungen stürzen. ${ }^{32}$ Sauerland meinte in seinem Gutachten, dass durch die Streichungen teilweise unvermittelte Übergänge entstehen mussten, die den aufmerksamen Leser befremden konnten. Die Widersprüche sollten vom Autor und dem Verlag überbrückt werden. Man könnte sie aber auch lassen, um den gewaltsamen Eingriff der Zensur fühlbar zu machen. Dadurch würde aber die künstlerische Qualität des Romans leiden. Die beiden Gutachter hatten sonst auch diverse stilistische Korrekturen vorgeschlagen.

Im Jahre 1970 trafen sich Grass und Wasilewski in Berlin und Warschau, um über die redaktionelle Bearbeitung der Blechtrommel zu beraten. Der Autor war nicht bereit, alle Streichungen zu akzeptieren. Offensichtlich ergaben sich für den polnischen Verlag unüberbrückbare Hürden, denn im April 1972 schrieb Wasilewski an Grass, dass die Veröffentlichung der polnischen Ausgabe verschoben werden müsse, da manche Probleme des Romans und ihre groteske, wenn auch höchst kunstvolle Vorstellung zu viele und zu große Meinungsunterschiede erwecken würden. ${ }^{33}$ Der Journalist Alexander Komitau, der in den 8oer Jahren den Gründen für die verspätete polnische Ausgabe der Blechtrommel nachgegangen war, kam zu der Erkenntnis, dass die Publikation des Romans von Jerzy Łukaszewicz 1971 gestoppt wurde ${ }^{34}$ (Łukaszewicz war in der regierenden Partei in Polen für die Presse und Propaganda zuständig).

Im Jahre 1979 wurde in Polen die Übersetzung der Blechtrommel in einer illegale Ausgabe publiziert, deren Auflagenhöhe etwa 2000 Exemplare betrug. Am 4. Oktober teilte Mirosław Chojecki, Leiter des unabhängigen Verlages

32 Grass (1987), 557 .

33 Andrzej Wasilewski an Grass, 22.04.1972, (AdK), Berlin, Günter-Grass-Archiv, Nr. 9451.

34 (AdK), Berlin, Günter-Grass-Archiv, Nr. 7265. 
NOWA, Günter Grass mit, dass knapp ein Jahr nach ihrer Unterhaltung in Danzig über die Veröffentlichung der Blechtrommel im Untergrund der Roman herausgekommen war. ${ }^{35}$ Chojecki bedankte sich bei dem Autor für die freundliche Genehmigung für den Druck und schrieb ihm, dass während der monatelangen Arbeit an der Buchausgabe die Materialien zweimal kurz vor einer Razzia in Sicherheit gebracht werden mussten. Da jedoch eine große Bindemaschine beschlagnahmt wurde, war der Roman nicht wie geplant in einem Band, sondern in zwei Heften erschienen. Nachdem der Roman im Untergrund herausgekommen war, wurde er 1983 auch vom Państwowy Instytut Wydawniczy herausgegeben. Dies hatte nun zur Folge, dass der Weg nach Polen für die Werke Grass' offen stand und in der nachfolgenden Zeit die Vorbereitungen für die Ausgabe von anderen Übersetzungen, nun im Danziger Verlag Wydawnictwo Morskie, getroffen wurden.

Der mit Grass befreundete Danziger Schriftsteller und Übersetzer seiner Lyrik, Bolesław Fac, resümierte während eines 1992 an der Universität Gdańsk gehaltenen Vortrags: „Deshalb wenn in der polnischen Öffentlichkeit Kontroversen um Grass ausgetragen wurden, ging es eben um dessen Polenbild [...]."36 Man kann Fac wohl zustimmen, denn seine Worte treffen weitgehend für die polnische Rezeption der Blechtrommel zu. Grass' Kritiker äußerten aber zum Teil ähnliche Bedenken, die nach dem Erscheinen des Romans auch in Deutschland laut wurden, wie die Vorwürfe der Blasphemie oder des Nihilismus. Die Abrechnung des Autors mit der jüngsten deutschen Geschichte und die gesellschaftliche Kritik wurden von der polnischen Literaturkritik eher wenig beachtet, dafür aber wurden diese Aspekte in den literaturwissenschaftlichen Abhandlungen dann desto intensiver erörtert.

\section{Literatur}

Błaut, S., „Polak tłumaczy Grassa“ [Ein Pole übersetzt Grass] in M. Janion (Hg.), Günter Grass i polski Pan Kichot (Gdańsk, 1999), 219-223.

Bratny, R., „Powitać piękną książkę“ [Wollen wir das schöne Buch begrüßen], in Polityka, July 17, 1971, 8.

Dobraczyński, J., „Obsesyjny infantylizm“ [Obsessiver Infantilismus], in Kierunki, September 8, 1963, 2 .

35 Mirosław Chojecki an Grass, 4.10.1979, (AdK), Berlin, Günter-Grass-Archiv, Nr. 9904.5.

36 Bolesław Fac, [Masch.], (AdK), Berlin, Günter-Grass-Archiv, Nr. 11123. 
Grass, G., „Dalekosiężna pieśń wyśpiewana ze szczytu wieży“ [Fernwirkender Gesang vom Stockturm aus gesungen. Übersetzt von Teresa Jętkiewicz], in Polityka, 51/52 (1958), 9 .

Grass, G. „Szeroka spódnica“ [Der weite Rock. Übersetzt von Teresa Jętkiewicz], in Nowa Kultura, 28 (1959), 4-5.

Grass, G., „Trybuna“ [Die Tribüne. Übersetzt von Teresa Jętkiewicz], in Nowa Kultura, 19 (1960), 5-6.

Grass, G., (1962a) „Poczta Polska“ [Die polnische Post. Übersetzt von Bolesław Fac], in Pomorze, 18 (1962), 3-4.

Grass, G., (1962b) „Poczta Polska“ [Die polnische Post. Übersetzt von Z.H.], in Kultura. Szkice. Opowiadania. Sprawozdania 4 (1962), 43-56.

Grass, G., „Zasnąłem pod czterema spódnicami“ [Ich schlief unter den vier Röcken ein], in Literatura na świecie, (1976), 3, 5-116.

Grass, G., Blaszany bębenek ([Warszawa], 1979).

Grass, G., Blaszany bębenek (Warszawa, 1983).

Grass, G. Die Blechtrommel (Göttingen, 1987 [1959]).

Hertz, P., „Rozważania o stanie i potrzebie tłumaczeń“, in Literatura na świecie, (1973), 12, 249-257.

Jaroszewski, M., „Der polnische Streit um Günter Grass' Blechtrommel“, in Studia Germanica Gedanensia, 10 (2002), 81-92.

Jeleński, K., „,Bęben‘ między Polską a Niemcami“ [Die ,Trommel` zwischen Polen und Deutschland], in Kultura. Szkice. Opowiadania. Sprawozdania, 1-2 (1962), 215-222.

Karaś, D., M. Wąs, in Zusammenarbeit mit I. Jopkiewicz, „Czy bohaterowie grywają w karty?" [Spielen die Helden Karten?], in Gazeta Wyborcza. Śledź te strony. Trójmiasto, December 11 2009, 14.

Koprowski, J. „Grass czy Graszewski“, in Tygodnik Kulturalny (1963), 47, 3, 6.

Koprowski, J., „Grass i polityka“ [Grass und die Politik], in Literatura na świecie, (1971), $12,323-326$.

Krauss, E., Roman Ingardens, Schematisierte Ansichten'und das Problem der Übersetzung: Die Blechtrommel von Günter Grass auf Polnisch (Berlin, 2011).

Misiorny, M., „Szeroki wybór i kodeks wymagań“ [Breite Auswahl und Moralkodex], in Trybuna Ludu, December 29, 1977, 5 .

Misiorny, M., „Pan K. uderza w bębenek“ [Herr K. rührt die Trommel], in Trybuna Ludu, January 15, 1984, 4.

N.N., „Marzenia są ciekawsze. Film o niezrealizowanych projektach Andrzeja Wajdy“ [Die Träume sind interessanter. Ein Film über die nicht verwirklichten Projekte von Andrzej Wajda], in Kino, 99 (1999), 25-29.

Ossowski, M., „Günter Grass i Andrzej Wajda. Wokół filmów, które nie powstały“ [Günter Grass und Andrzej Wajda. Zu den Filmen, die nicht entstanden sind], in 
G. Jaśkiewicz/J. Wolski (Hg.), Literatura lekturą i doświadczeniem próbowana czyli Zbigniewa Świattowskiego germanistyka prometejska (Rzeszów, 2013), 201-214.

Zielińska, M., „W ślimaczym tempie ... Uwarunkowania polskiej recepcji literatury niemieckiej na przykładzie debat wokół twórczości Güntera Grassa w wybranych czasopismach literackich i społeczno-kulturalnych (1961-1981)“ [Im Schneckentempo ... Die Bedingungen der polnischen Rezeption von der deutschen Literatur am Beispiel der Debatte um das Werk von Günter Grass in ausgewählten literarischen und gesellschaftlich-kulturellen Zeitschriften (1961-1981)], in A. Zawiszewska/ A. Borkowska (Hg.), Nie tylko Zachód. Recepcja literatur obcych w czasopismach polskich XX wieku (Łask, 2006), 61-78, 62-63.

Zwarra, B., Gdańsk 1939. Wspomnienia Polaków-Gdańszczan [Danzig 1939. Erinnerungen der Danziger Polen]. Ausgewählt und eingeleitet v. B. Zwarra (Gdańsk, 1984).

Żukrowski, W., „Bełtanie w głowie“ [Quirlen im Kopf], in Kultura, October 6, 1963, 12.

Żyliński, L., „Die Eigenart der polnischen Rezeption von Günter Grass“, in Oldenburger Universitätsreden. Vorträge. Ansprachen. Aufsätze (Oldenburg, 2009). 